\title{
A counterexample to a conjecture of Erdős, Graham and Spencer
}

\author{
Song Guo* \\ Department of Mathematics, Huaiyin Teachers College, \\ Huaian 223300, The People's Republic of China \\ guosong77@hytc.edu.cn
}

Submitted: Oct 6, 2008; Accepted: Dec 2, 2008; Published: Dec 9, 2008

Mathematics Subject Classification: 11B75

\begin{abstract}
It is conjectured by Erdős, Graham and Spencer that if $1 \leq a_{1} \leq a_{2} \leq \cdots \leq a_{s}$ with $\sum_{i=1}^{s} 1 / a_{i}<n-1 / 30$, then this sum can be decomposed into $n$ parts so that all partial sums are $\leq 1$. In this note we propose a counterexample which gives a negative answer to this conjecture.
\end{abstract}

Keywords: Erdős-Graham-Spencer conjecture; Erdős problem; Partition.

\section{Introduction}

Erdös ([2], p. 41) asked the following question: is it true that if $a_{i}$ 's are positive integers with $1<a_{1}<a_{2}<\cdots<a_{s}$ and $\sum_{i=1}^{s} 1 / a_{i}<2$, then there exists a subset $\mathrm{A}$ of $\{1,2, \ldots, s\}$ such that

$$
\sum_{i \in A} \frac{1}{a_{i}}<1, \sum_{i \in\{1, \ldots, s\} \backslash A} \frac{1}{a_{i}}<1 ?
$$

Sándor [3] gave a simple construction to show that the answer is negative: let $\left\{a_{i}\right\}=$ \{ divisors of 120 with the exception of 1 and 120$\}$. Furthermore, Sándor[3] proved the following results:

Theorem 1. For every $n \geq 2$, there exist integers $1<a_{1}<a_{2}<\cdots<a_{s}$ with $\sum_{i=1}^{s} 1 / a_{i}<n$ and this sum cannot be split into $n$ parts so that all partial sums are $\leq 1$.

${ }^{*}$ This author is supported by Natural Science Research Project of Ordinary Universities in Jiangsu Province (08KJB110002),P.R.China. 
Theorem 2. Let $n \geq 2$. If $1<a_{1}<a_{2}<\cdots<a_{s}$ with $\sum_{i=1}^{s} 1 / a_{i}<n-\frac{n}{e^{n-1}}$, then this sum can be decomposed into $n$ parts so that all partial sums are $\leq 1$.

If we allow repetition of integers, it is conjectured by Erdös, Graham and Spencer ([2],p. 41) that if $1 \leq a_{1} \leq a_{2} \leq \cdots \leq a_{s}$ with $\sum_{i=1}^{s} 1 / a_{i}<n-1 / 30$, then this sum can be decomposed into $n$ parts so that all partial sums are $\leq 1$. This is not true for $\sum_{i=1}^{s} 1 / a_{i} \leq n-1 / 30$ as shown by $a_{1}=2, a_{2}=a_{3}=3, a_{4}=\ldots=a_{5 n-3}=5$. Sándor[3] proved a weaker assertion when the $n-1 / 30$ was replaced by $n-1 / 2$.

Let $\alpha(n)$ denote the least real number such that: for any integers $1 \leq a_{1} \leq a_{2} \leq \cdots \leq$ $a_{s}$ with $n \geq 2$ and $\sum_{i=1}^{s} 1 / a_{i}<n-\alpha(n)$, this sum can be decomposed into $n$ parts so that all partial sums are $\leq 1$. Erdős-Graham-Spencer conjecture hoped that $\alpha(n) \leq 1 / 30$ and Sándor's result stated that $\alpha(n) \leq 1 / 2$. In [1] Yong-Gao Chen proved that $\alpha(n) \leq 1 / 3$ and in [4] Jin-Hui Fang and Yong-Gao Chen proved that $\alpha(n) \leq 2 / 7$.

The purpose of this article is to give a counterexample to Erdős-Graham-Spencer conjecture:

$$
a_{1}=2, a_{2}=a_{3}=3, a_{4}=4, a_{5}=\cdots=a_{11 n-12}=11,
$$

which stats that

Theorem 3. $\alpha(n) \geq 5 / 132$.

\section{Proof of Theorem 3}

Clearly,

$$
\sum_{i=1}^{11 n-12} \frac{1}{a_{i}}=n-\frac{5}{132}
$$

For any partition $\{1, \ldots, 11 n-12\}=\cup_{j=1}^{n} A_{j}$, we will prove that there exists $1 \leq j \leq n$ such that $\sum_{k \in A_{j}} 1 / a_{k}>1$. Without loss of generality, we let $1 \in A_{1}$. Let $l=\mid A_{1} \cap$ $\{2,3,4\} \mid$. Below we distinguish four cases.

Case 1. $l \geq 2$.

In this case we must have

$$
\sum_{k \in A_{1}} \frac{1}{a_{k}} \geq \frac{1}{2}+\frac{1}{3}+\frac{1}{4}=\frac{13}{12}>1
$$

and we are done.

Case 2. $l=1$ and $4 \notin A_{1}$.

Assume that $t \in \mathbb{N}$ and

$$
\sum_{k \in A_{1}} \frac{1}{a_{k}}=\frac{1}{2}+\frac{1}{3}+\frac{t}{11}
$$

If $t \geq 2$, we have

$$
\sum_{k \in A_{1}} \frac{1}{a_{k}} \geq \frac{1}{2}+\frac{1}{3}+\frac{2}{11}=\frac{134}{132}>1
$$


If $0 \leq t \leq 1$, we must have

$$
\sum_{j=2}^{n} \sum_{k \in A_{j}} \frac{1}{a_{k}} \geq n-\frac{5}{132}-\left(\frac{1}{2}+\frac{1}{3}+\frac{1}{11}\right)=n-1+\frac{5}{132}>n-1 .
$$

Thus there exists $2 \leq j \leq n$ such that $\sum_{k \in A_{j}} 1 / a_{k}>1$ and we are done.

Case 3. $l=1$ and $4 \in A_{1}$.

Assume that

$$
\sum_{k \in A_{1}} \frac{1}{a_{k}}=\frac{1}{2}+\frac{1}{4}+\frac{t}{11}
$$

One can see that

$$
\sum_{k \in A_{1}} \frac{1}{a_{k}}=\frac{1}{2}+\frac{1}{4}+\frac{3}{11}=\frac{135}{132}>1
$$

when $t \geq 3$ and

$$
\sum_{j=2}^{n} \sum_{k \in A_{j}} \frac{1}{a_{k}} \geq n-\frac{5}{132}-\left(\frac{1}{2}+\frac{1}{4}+\frac{2}{11}\right)=n-1+\frac{4}{132}>n-1,
$$

hence there exists $2 \leq j \leq n$ such that $\sum_{k \in A_{j}} 1 / a_{k}>1$ when $t \leq 2$. So we prove it.

Case 4. $l=0$.

Assume that

$$
\sum_{k \in A_{1}} \frac{1}{a_{k}}=\frac{1}{2}+\frac{t}{11} \leq 1
$$

Then we have $t \leq \frac{11}{2}$ and hence $t \leq 5$. Thus we conclude that

$$
\sum_{j=2}^{n} \sum_{k \in A_{j}} \frac{1}{a_{k}} \geq \frac{2}{3}+\frac{1}{4}+\frac{11 n-21}{11}=n-1+\frac{1}{132}>n-1,
$$

and hence there exists $2 \leq j \leq n$ with $\sum_{k \in A_{j}} 1 / a_{k}>1$. Now we complete the proof.

Acknowledgment. The author acknowledge professor Zhi-wei Sun for introducing this subject and the referee for his/her helpful suggestions.

\section{References}

[1] Yong-Gao Chen, On a conjecture of Erdös, Graham and Spencer, J. Number Theory 119 (2006) 307-314.

[2] P. Erdős, R.L. Graham, Old and New Problems and Results in Combinatorial Number Theory, Enseign. Math. (2), vol. 28, Enseignement Math., Geneva, 1980.

[3] C. Sándor, On a problem of Erdős, J. Number Theory 63 (1997) 203-210.

[4] Jin-Hui Fang and Yong-Gao Chen, On a conjecture of Erdős, Graham and Spencer, II, Discrete Appl. Math., 156(2008) 2950-2958. 